\title{
Hybrid IR FPAs based on charge injection devices
}

\author{
V.M. Bazovkin, N.A. Valisheva, A.A. Guzev, V.M. Efimov, A.P. Kovchavtzev, \\ G.L. Kuryshev, I.I. Lee, I.V. Mzhelskiy, V.G. Polovinkin, A.V. Zarenko.
}
Rzhanov Institute of Semiconductor Physics, Siberian Branch of the Russian Academy of Sciences, e-mail irlamlee@isp.nsc.ru

The points of structuring hybrid IR FPAs based on charge injection devices (CID photodetector) are considered. Results of experimental examination of different thermographic systems based on InAs CID, linear and matrix hybrid modules, IR camera, IR microscope, high speed spectrometer are presented. It is shown that, in the short-wave IR range, it is possible to realize thermographic systems with temperature resolution (4-8) $\mathrm{mK}$ at effective frame frequency $1-10 \mathrm{~Hz}$.

Key words: IR focal plane arrays, CID photodetector, read-out circuit (ROIC).

\section{Introduction}

Characteristics of CID-based hybrid IR FPAs are reported. An advatage of CID elements in comparison with other IR photodetector types (photodiodes, photodetectors based on multi-layer quantum wells) is the possibility to integrate photosignals directly in the CID element. In this case, it is not necessary to have the accumulative element in the input cell and the requirements for the ROIC become much less stringent.

In $\S 2$ the analysis temperature resolution of thermographic systems (NETD), depending on the spectral range and read-out circuits charge capacity is formulated. In $\S 3$ ways of photosignals read-out from multielement CIDs and basic parameters of hybrid photodetector modules are considered. In $\S 4$ and 5, there is a description of silicon read-out circuits from multi-element CID photodetectors and the basic parameters of hybrid IR FPA modules. In $\S 6$ characteristics of thermography systems based on matrix hybrid module on InAs, high speed IR spectrometer based on the linear module formatted 1x384 within selection time $0.1-10 \mathrm{~ms}$, scanning IR microscope are given.

\section{Analysis of thermovision systems temperature resolution depending on read-out circuits spectral range and charge capacity.}

Analysis noise equivalent temperature difference (NETD) of thermographic systems depending on spectral range and charge capacity of ROIC was carried out in a number of papers [1-5]. In Fig. 1 numeric NETD calculations depending on spectral range and charge capacity at room background temperature $300 \mathrm{~K}$ [2] are presented. 
In Fig. 1, curve 1 shows the well known spectral dependencies of NETD for thermographic systems with IR FPA photosensitive channels operate in BLIP mode and the charge capacity of read out circuit is sufficiently high for the photosygnals to be accumulqated over the whole frame time of $20 \mathrm{~ms}$. The character of dependencies cardinally changes if it is estimated at limited charge capacity read-out circuits. Charge capacity of electrons $2 \cdot 10^{6}$ (curve 2, Fig. 1) corresponds to InAs CID elements capacity [6]. Charge capacity $210^{7}$ of electrons (curve 3 ) is typical of silicon-based read-out devices of matrix IR FPAs based on photodiodes with spacing 20-30 $\mu \mathrm{m}$ [7].

In the limited conditions of charge capacity ROIC, NETD decreasing with $\lambda$ growth, and the best NETD is achieved in the spectral range at which the background radiation level corresponds to read-out device charge capacity (or CID element's charge capacity) at the time of photosignals accumulation equal to that of frame time. As CID elements charge capacity decreases in the regime of non-equilibrium depletion on narrow-band, therefore, for room background temperatures, this condition is observed in rather a narrow spectral range of $2.8-3.5 \mu \mathrm{m}$.

\section{Principles of photosignal read-out from CID photodetectors.}

Some circuit-technical solutions used for signals read-out from photodiodes can be applied in hybrid IR CID-based FPA [8, 9]. In Fig.2, schemes of photosignals read-outs from photodiodes and CID elements are shown.

The disadvantage of the potential readout from CID elements, compared to the readout from photodiodes, is that the ratio of photosignal conversion (a photosignal for CID element is the charge of the surface Ys) to voltage at the amplifier input decreases proportionally to the ratio of capacities $\mathrm{C}_{0} /\left(\mathrm{C}_{0}+\mathrm{C}_{\mathrm{D}}\right)$, where $\mathrm{C}_{\mathrm{D}}$ is capacity of nonequilibrium depletion area of CID element, $\mathrm{C}_{0}$ - gate value of CID element. Hence, the response of the IR FPA based on CID elements with potential read out circuits is lower than that of IR FPA based on photodiodes. Moreover, for reading out from CID elements to the drain of transistor T1 during applying the pulse voltage $F_{c}$, we have to apply not the constant voltage but the injection pulse $F_{\text {inj }}$ that is required for restoring the mode of nonequilibrium depletion of CID element in each readout cycle. The injection pulse $F_{\text {inj }}$ can be applied on the substrate $U_{\text {sub }}$ of CID element, in this case, the constant bias supply voltage $\mathrm{U}_{\mathrm{b}}$ is applied on the drain transistor T1 (fig.2b), the control voltages are given in brackets.

To read out photosignals from CID photodetectors, it is also possible to use direct charge injection read-out circuits, Fig.3.

The difference of direct injection circuit from CID elements with the same read-out photodiode circuit is introduction of the additional diffusion area 5, that of charge-connected with 
accumulation gate 3 through gate 4 and transistor T1. These additional elements are necessary to set the CID element to the regime of non-equilibrium mode and attachment of the potential on CID element gate to the surface potential under the input gate of read-out circuit before each accumulation cycle. Herein, substrates of a CID element and silicon read-out circuits should be of one type ( $\mathrm{n}$ or $\mathrm{p}$ ). When supplying injection pulse Finj, minor carriers accumulated in the previous cycle recombinate in substrate with major carriers and the non-equilibrium depletion mode regenerates after the end of Finj. Accumulation of photogenerated carriers in the inverse layer leads to an increase of a CID element's capacity. Therefore, after the end of impulse voltage Fres excessive charge proportional to photocurrent integrates under the accumulation gate 3 . To optimize the operating regime of such a device, the time gap between the ends of impulses Finj, Fc and duration of pulse Fres is of principal importance. These time intervals are to be sufficient so that, by the moment of pulse Fres completion, the potential at which the current integrated in the input device becomes close to the photocurrent level in the CID detector on the input diffusion area 1. Current input coefficient $\left(\eta_{\mathrm{I}}\right)$ from the CID element into the input circuit with direct charge injection - unlike analogous dependencies when reading photodiode signals - does not, practically, depend on the voltage on the input gate Uin [10]. For this reason, for CID elementbased IR FPA, requirements to the scattering value of threshold voltages become lower (less strict) and, hence, the level of geometric noises also becomes lower.

The possibility of accumulating photosignals directly in the CID element is an important advantage of CID elements. In this case, there is no necessity of placing an accumulative element in the read-out device and it makes the requirements for pre-amplifier considerably lower. The scheme of photosignals read-out device with intrinsic accumulation in the CID element is presented in Fig. 4 [8].

As this read out circuit if a CID element's substrate is of n-type, it is necessary to use a ptype substrate for a silicon read-out circuit and to realize the device using the simplest n-channel technology. When supplying injection impulse Finj through dividing capacity Cinj, in the interval of pulse Fres, the minor carriers accumulated in the inverse layer recombine in the CID element's capacity with the major ones. By the end of pulse Finj, CID photodetectors are again set in the non-equilibrium mode. With the switched on pulse Fres, the potential on the CID element's gate again binds with that under the input gate, the excessive charge is with drawn to the source of supply. After the end of Fres pulse, the charge proportional to the photosignal charge accumulated in the inverse area flows down from the CID element along the circuit. Input diffusion area 1, charge-bound gates 2 and 3 integrate under accumulation gate 4 . It is important to note that the 
CID element's binding to surface potential under the input gate of the read-out circuit before and after the supply of injection Finj pulse, i.e. a correlated sampling and, thus, low-frequency noise components of a read-out circuits are suppressed.

\section{Hybrid matrix CID element-based IR FPAs}

A principle circuit of multiplexor for 2D matrix IR FPA is shown in Fig. 5. The operation of read-out circuit is explained by tme diagrams of control voltages, Fig. $5 \mathrm{~b}$.

The multiplexor is fabricated on n-channel technology with two levels of polysilicon gates. Maximal charge capacity is provided by a maintenance of constant potential on the CID element gate. This circuit allows us to bring about accumulation time regulation within $20 \mathrm{~ms}$ to the frame time. Time regulation is provided by a successive introduction of two control buses Finj1 and Finj2 with a period of 4 lines, impulses Fin"a" and Fin"b" are applied. In case of the necessity for accumulation time regulation on the vertical shift register input F0v, the second logical "1" is supplied with a delay multiple to 2 rows duration. In this case, with the movement of the second logical "1", in the vertical shift register, pulse Fin"b" is supplied to dividing capacity, and the frame time is divided into two parts: one part is used for signal charge accumulation which then goes to pre-amplifiers inputs; the other part of the frame is connected with charge accumulation which is not used and it is with drawn to the supply source.

MIS structures were fabricated on (111A) oriented (ESAI-40-35-10 and ESAI-92-35-10 Yae $0.040 \mathrm{Tu})$ InAs epitaxial structures, charge carriers concentration in the epitaxial layer $(1 \div 3) \cdot 10^{15}$ $\mathrm{cm}^{-3}$, mobility $(8 \div 12) \cdot 10^{4} \mathrm{~cm}^{2} \mathrm{~V}^{-1} \mathrm{~s}^{-1}$, lifetime of minority carriers $(0.3-1.8) \mu \mathrm{s}$.A fabrication technology of CID elements providing high stability of electrophysical and photoelectric characteristics has been developed. So, surface state density is less than $2 \cdot 10^{10} \mathrm{~cm}^{-2} \mathrm{eV}^{-1}$, charge density of 'slow' states is not more than $5 \cdot 10^{-8} \mathrm{~K} / \mathrm{cm}^{2}$, electric field breakdown intensity of the gate dielectric is $(4 \div 6) 10^{6} \mathrm{Vcm}^{-1}[8]$.

For 2D hybrid modules based on InAs CID elements, radiation from the object under study is incident on the non-planar side of a strongly doped wafer $\mathrm{n}^{++}$-InAs. Due to a shift of fundamental absorption edge (Moss-Burstein effect), radiation transmits only in the spectral range $\sim 2,4-2,5 \mu \mathrm{m}$ and depends on the level of doping the degenerated InAs substrate. Long-wave edge $(\lambda \sim 3.05 \mu \mathrm{m})$ is determined by the fundamental absorption edge in epitaxial $n$-InAs layer.

Detectivity D* of a hybrid IR FPA formatted $128 \times 128$, at the temperature $77 \mathrm{~K}$, is $\sim 3.310^{12}$ $\mathrm{cmHz}^{1 / 2} \mathrm{~W}^{-1}$ (at background radiation level $\sim 6,410^{-7} \mathrm{Wcm}^{-2}$ ) which corresponds to the sensitivity in BLIP mode for the quantum efficiency of CID photodetectors $=0.7$. Photoelectric relationship 
among neighboring elements does not exceed $2 \%$ [9].

\section{Linear-type hybrid IR FPAs.}

A silicon multiplexor of 2x192 format [9] has been developed for the linear type IR FPA. It has two mirror-symmetric 192 input multiplexors with spacing $50 \mu \mathrm{m}$ shifted at $25 \mu \mathrm{m}$ related to each other. The principle circuit of read-out devices is close to that of matrix multiplexor shown in Fig 5. The main parameters of silicon multiplexor are the number of input devices 2x192, level of intrinsic noise for 400-600 electrons, pixel rate to $5 \mathrm{MHz}$, regulated accumulation time, minimal (from $30 \mu \mathrm{s}$ ); consumed capacity $-50 \mathrm{~mW}$, number of information outputs -2 ; dynamic range not less than $75 \mathrm{~dB}$.

Construction of multiplexor permits the possibility to realize two variants of the hybrid circuit. In the first variant, the photodetector line crystal is placed between inputs of two mirrorsymmetrical multiplexors and electrically binds with them with In bumps, 5-7 $\mu \mathrm{m}$ high, using the flip-chip method. Photosensitive elements are illuminated through preliminary antireflective nonplanar side of multiplexor silicon wafer which is a cut-out filter and it forms photodetector elements $(\sim 1.1 \mu \mathrm{m})$ short-wave sensitivity edge. Long-wave sensitivity edge $(3,05 \mu \mathrm{m})$ is determined by the absorption edge of InAs. The photosensitive line has a chess-like elements location and spacing equal to $50 \mu \mathrm{m}$, CID gate size is $26 \times 26 \mu \mathrm{m}$, gaps between photodetectors are protected with a metal screen (Ti).

In the second variant, the Si multiplexor is divided into two separate crystals. The optical signal is projected directly on photodetector elements and realization of spectral range 0.5-3.05 $\mu \mathrm{m}$ becomes possible. Hybrid modules are realized in the construction to solve spectroscopic tasks. One-row photodetectors line sized 1x384 with spacing $25 \mu \mathrm{m}$ is used in these devices. Practically all CID elements are functional in the BLIP mode at background level $\sim 1,710^{-7} \mathrm{~W} \mathrm{~cm}^{-2}$.

\section{Thermovision systems based on multi-element InAs CID elements.}

\subsection{Thermographic system TKVr-ISP "SVIT"}

The hybrid module of $128 \times 128$ format is installed into a fill-in ivacuum qryostat with a carbon getter.

Specification of IR camera TKVr-ISP "SVIT":

Lens..............................

Photosensitive element size, $\mu \mathrm{m} . . . \ldots \ldots \ldots \ldots \ldots \ldots \ldots . . . . . .40 \times 40$;

Spectral sensitivity range........................2,4-3,05 $\mu \mathrm{m}$;

Frames frequency, fr , to.........................200 $\mathrm{c}^{-1}$;

Temperature resolution, NETD $\left(\mathrm{fr}=100 \mathrm{c}-1, \mathrm{~T}=320^{\circ} \mathrm{C}\right) \ldots \ldots \ldots \ldots . . .0,0250 \mathrm{C}$;

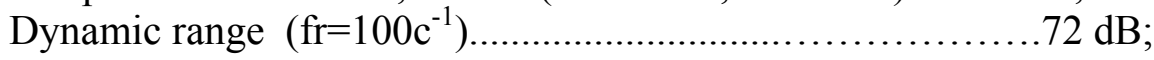


Absolute precision of temperature measurement at system temperature $22 \pm 20{ }^{\circ} \mathrm{C}$ $. \pm 0,25{ }^{0} \mathrm{C}$;

Supply from USB2.0 computer port, consumed by system, $1.1 \mathrm{~W}$;

Cooling agent liquid nitrogen, hours

time of continuous operation without liquid nitrogen ad-pouring, 10-12

The temperature range is $10 \div 40^{\circ} \mathrm{C}$ in the device calibration for medical application. The device may be calibrated to $200^{\circ} \mathrm{C}$ by means of accumulation time increase and higher when using grey filters to study high-temperature objects.

High quality of CID elements on InAs and the developed read-out circuits provided lower 1/f-type noises by photosignals summing from several frames, a considerable increase of TKVr-ISP "SVIT" temperature resolution. Experimental dependencies of temperature resolution from the effective frame frequency at the temperature of scene $30^{\circ} \mathrm{C}$ are presented in Fig. 6, where $\mathrm{N}$ is summed frames number, and the effective frame frequency is determined on the ratio of 100 frames $\mathrm{c}^{-1} / \mathrm{N}$. From the picture it is seen that $\operatorname{NETD}(\mathrm{N})$ are quite close to $1 / \mathrm{N}^{1 / 2}$ (curve $\mathrm{C}$ in Fig. 6) up to 128 accumulation cycles, $\mathrm{NETD}(\mathrm{N})$ is improved from $25 \mathrm{mK}$ at one accumulation cycle to $3-4 \mathrm{mK}$ at 128 accumulation cycles.

In our opinion, the spectral range $\sim 3 \mu \mathrm{m}$ is optimal for medico-biological applications, At room temperature of the background and objects temperature $30-35^{0} \mathrm{C}$, in this spectral range, the background radiation level is 2-3 orders of value lower, and the image contrast is 2-3 times higher compared to spectral range 5-12 $\mu \mathrm{m}$.

Due to availability and simplicity in exploitation of modern thermographic systems, absence of hazardous factors, setting effective thermovisional methods aimed at human health control, thermovision is becoming one of the most important diagnostic methods in medicine. IR camera TKVr-ISP "SVIT" is certified by RF Healthcare Ministry, European certificate (EC Certificate No110176QS/NB on 27th February, 2011) and it is widely used in scientific investigations, e.g. in studying latent defects of multilayer semiconductor structures obtained by silicon direct bonding method [12], thermal exchange in liquid media [13].

\subsection{Infrared microscopes}

The IR microscope is produced on the base of hybrid module formatted $128 \times 128$. In an infrared microscope, it is important to provide background radiation screening. In our device it is realized using a mirror screen which allows us to reduce background radiation to the value equivalent to spatiol angle $\sim 0.009$ comparable to the objective's corporal angle. To build up an 
object's image, a lens objective with focal distance $20.5 \mathrm{~mm}$ and relative orifice 1:1.8 is used. The objective carries out diffraction resolution limit $~ 3.0 \mu \mathrm{m}[14,15]$.

NETD dependencies from accumulation time and an object's temperature as a parameter are presented in Fig. 7. As is seen from the diagram, NETD is improved with an increase of accumulation time and temperature of test objects. The above-described image of hybrid module formatted 2x192, obtained by a scanning IR microscope from the non-planar lit side of the silicon-based multiplexor is presented in Fig 8. Hidden images of topology elements on the planar side of the silicon multiplexor and line array InAs photosensitivity elements on, assembled on In microbumps with flip-chip method, are seen.

\subsection{High speed spectrometer}

The high speed spectrometer for spectral range 1-3 $\mu \mathrm{m}$ based on InAs of CID elements and spectral range 1-5.4 $\mu \mathrm{m}$ based on InSb of CID elements [9] was developed using the hybrid module of format $1 \times 384$. The spectrometer consists of a hybrid module installed in vacuum cryostat and of monochromator MS2004I. Monochromator MS2004I of brand Solar TII (Minsk, Belarus) is an automatic device, PC-controller via interface RS232. When dealing with difraction lattice $400 \mathrm{u} / \mathrm{mm}$, it provides with spectral scannings within 1135-3400 nm. Inverse linear dispersion on the output of monochromator is $\sim 12 \mathrm{~nm} / \mathrm{mm}$ which allows spectral resolution $0.3 \mathrm{~nm}$ per element. The spectrometer provides spectral registration within time intervals $0.1-10 \mathrm{~ms}$.

\section{Conclusion}

Developments and investigations of hybrid multielement IR FPAs and thermographic systems based on InAs elements of CID are presented. High temporal stability of the elements we developed of InAs-based CID elements of thermographic systems for various application (IR camera, high speed IR spectrometer, scaning IR microscope) provides a considerable increase of their sensitivity by means of frames summing. Thus, for instance, at efficient frame frequency $\sim 1 / \mathrm{s}$, the temperature resolution of TKVr-ISP "SVIT" camera is $\sim 4 \mathrm{mK}$, i.e. higher than the thermographic systems known to us in the long-wave spectral range.

\section{References}

1. I.I. Taubkin, M.A Trishenkov, "Minimum temperature difference resolvable with the IR imaging method", Opticheskii Zhurnal, 5, 20-23, (1993).

2. I.I. Lee, "Dependence of the Noise Equivalent Temperature Difference of Thermal Imaging 
Systems on the Readout Circuit Charge-Handling Capacity," Optoelectronics, Instrumentation and Data Processing, No. 2, 114 (2001).

3. Tribolet P., Chorier P., Manissandrian A. "High performance infrared detectors at Softradir". SPIE, Vol.4028, p.438-455, (2000).

4. V.A.Markin, "Temperature -sensitivity limits of the third-generation thermal viewers", Journal of Optical Technology", Vol.77, I. 2, pp.119-125, (2010).

5. J.V. Gumenjuk-Sichevska, D.D. Karnaushenko, I.I. Lee, and V.G. Polovinkin, "Infrared photodetectors based on the system $\mathrm{Hg}_{1-\mathrm{X}} \mathrm{Cd}_{\mathrm{X}} \mathrm{Te}$ photodiode - direct-injection readout circuit". Optoelectronics review, 19, no. 2, p. 63-70, (2011).

6. A. Rogasky. Infrared Detectors, Gordon and Breach Science Publishers, Amsterdam, 2000.

7. Charge-Coupled Devices, Ed. by D.F.Barbe, Springer, Berlin, 1980.

8. I.I. Lee, V.M. Bazovkin et al., "Multielement hybrid IR FPA based on charge-injected devices: Part I. Principles of signal readout". Optoelectronics, instrumentation and data processing, 43, Nomb.4, 314-322 (2007).

9. I.I. Lee, V.M. Bazovkin et al., "Multielement hybrid IR FPA based on charge-injected devices: Part II. Thermography systems with InAs elements". Optoelectronics, instrumentation and data processing, 43, no.4, 322-331, (2007).

10. Woolaway J., "New sensor technology for the 3-to5 $\mu \mathrm{m}$ imaging band", Photonics spectra, p.113, (February 1991).

11. B. Vainer, "FPA-based infrared thermography as applied to the study of cutaneous perspiration and stimulated vascular response in human”, Physics in Medicine and Biology, 50), 63-94, (2005).

12. B.G. Vainer, G.N. Kamaev, G.L. Kuryshev, "Application of the narrow spectral range InAsFPA-based IR camera for the investigation of the interface voids in silicon wafer bonding", J. Cryst. Growth, 210, 1-3, p.351-355, (2000).

13. V.S. Berdnikov, V.A. Grishkov, V.A. Markov, Yu.M. Pshenichnikov, "Thermovision research of thermal gravitational-capillary convection in a horizontal layer heated from below", International Conference "Advanced problems in thermal convection", Perm-2003, 24-27, 27-28, (November, 2003).

14. V. M. Bazovkin, I. V. Mzhel'skiy, G. L. Kuryshev, and V. G. Polovinkin, "Infrared Scanning Microscope with High Spatial Resolution", Optoelectronics, Instrumentation and date processing, 47, no. 5, 498-502, (2011).

15. G. L. Kuryshev, I. V. Mzhel'skiy, A. E. Nastov'yak, and V. G. Polovinkin, “Calibration of Photodetectors in IR Microscopes”, Optoelectronics, instrumentation and data processing, 47, no. 
5, 498-502, (2011).

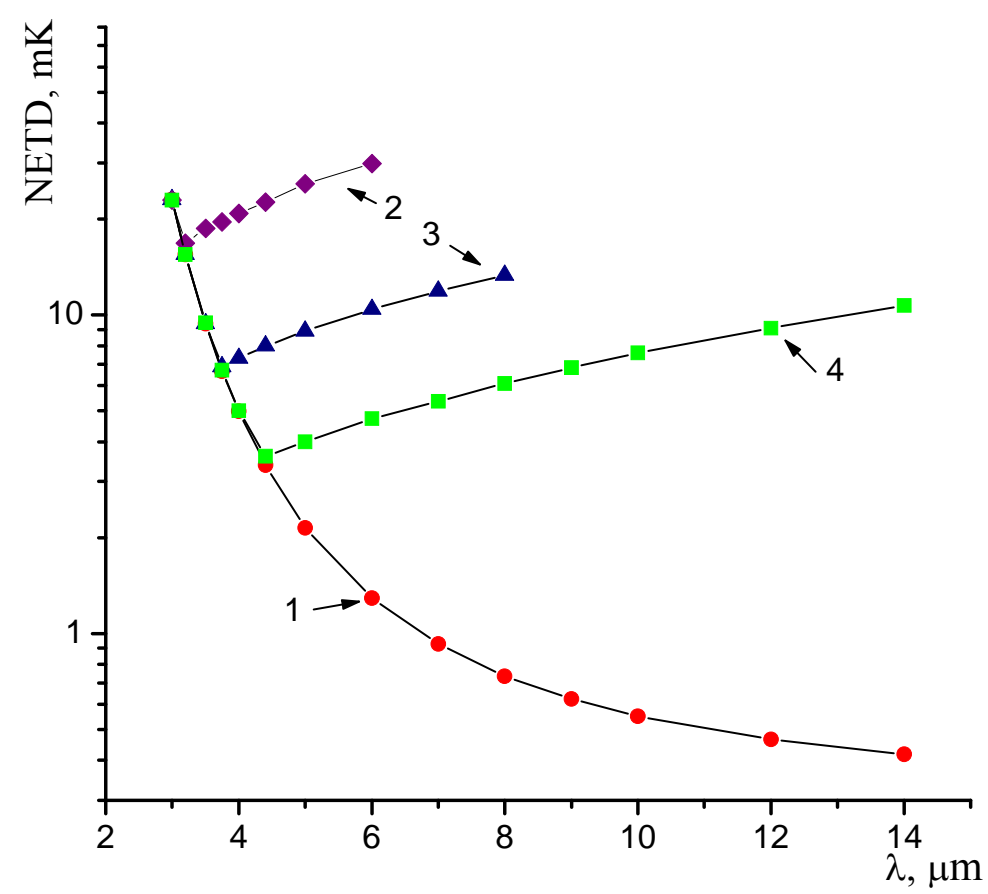

Fig. 1. Calculative NETD dependencies on the wavelength and charge capacity of the readout device as a parameter, at background temperature $=300 \mathrm{~K}$. Curve 1 at aperture $2 \mathrm{~F} / \mathrm{D}=3$. Accumulation time is equal to frame time $(20 \mathrm{~ms})$. For curves 2-4, NETD was calculated provided at limited charge capacity of the read-out device: curve $2-210^{6}$, curve $3-2$ $10^{7}$ and curve $4-810^{7}$ electrons, photodetector area $-710^{-5} \mathrm{~cm}^{2}$.
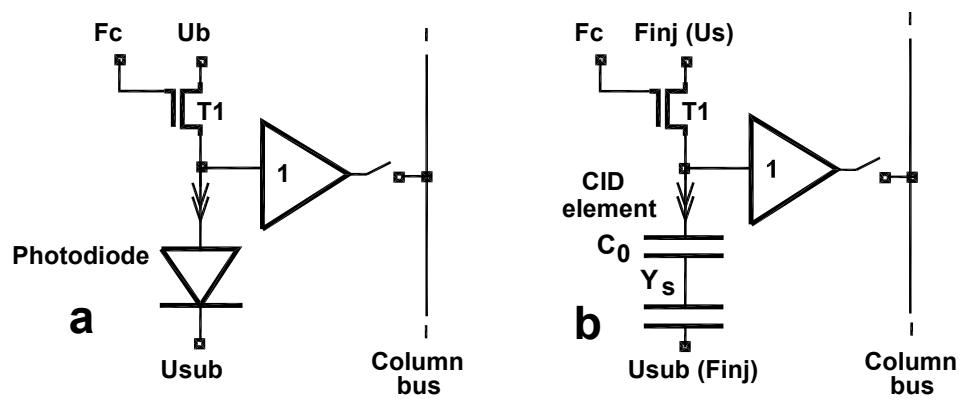

Fig. 2. A diagram of the circuit with potential readout and integration of photosygnals in photodiode (a), CID photodetector (b). 


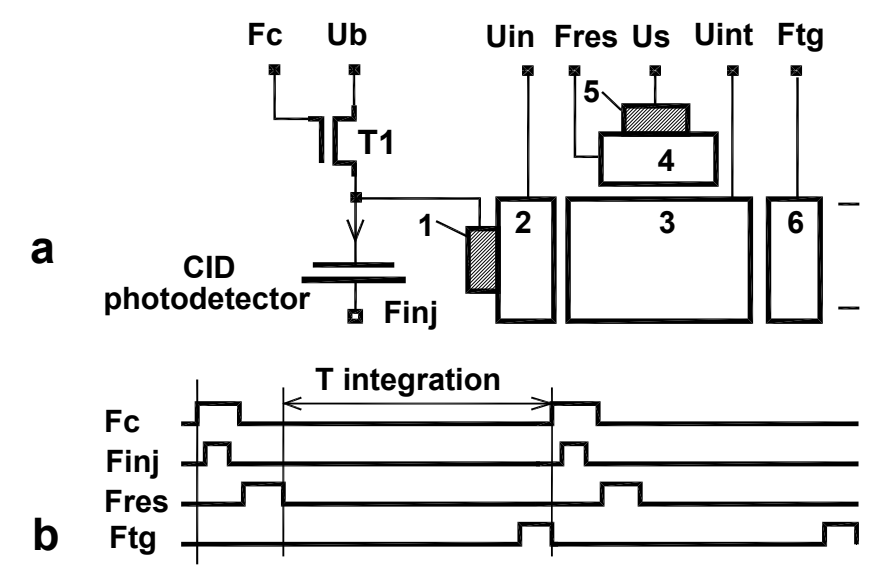

Fig. 3. Read-out system; CID photodetector - a direct-injection circuit: principal cell circuit (a), time diagrams of controlling voltages (b).

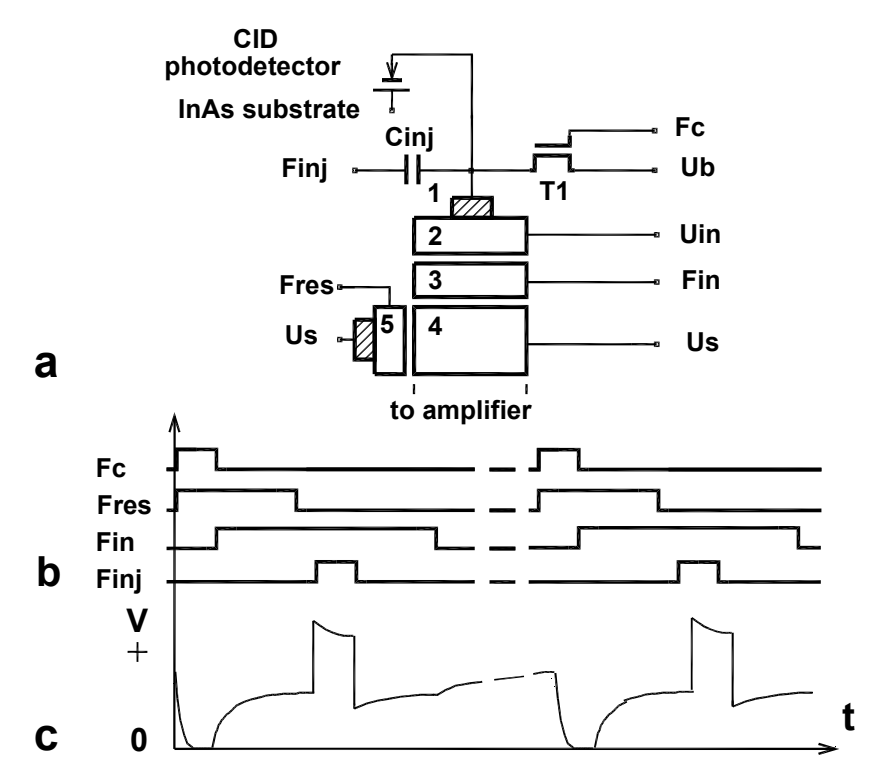

Fig. 4. Principle of signal read-out from CID elements with intrinsic acccumulation, where a — principle circuit of silicon multiplexor, b - plots of control voltages, $\mathrm{c}$ - plots of voltages on CID element gate. 

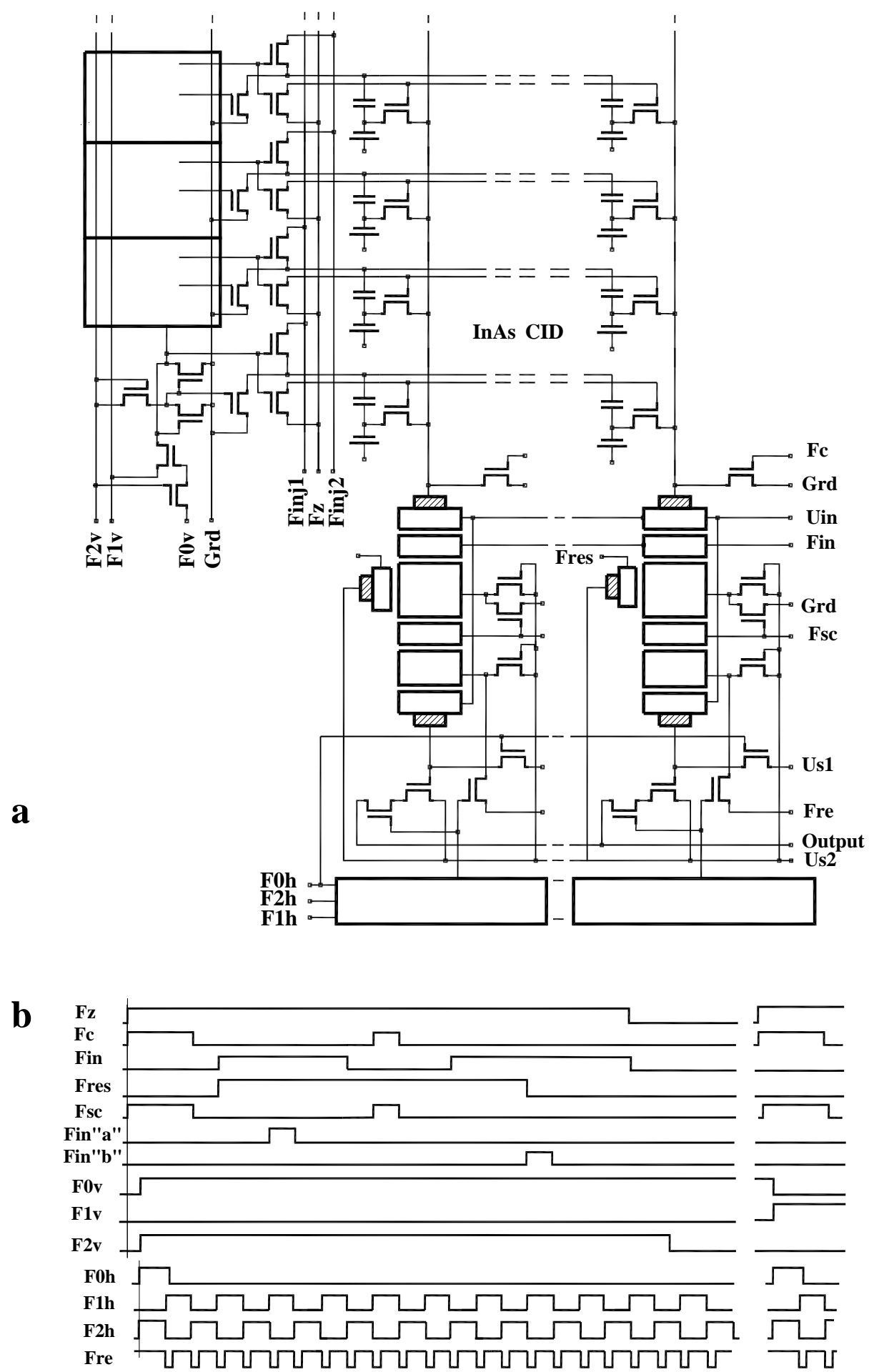

Fig. 5. Principle circuit of matrix silicon multiplexor for photosignals read-out from CID elements. (a); time diagrams of controlling voltages (b). 


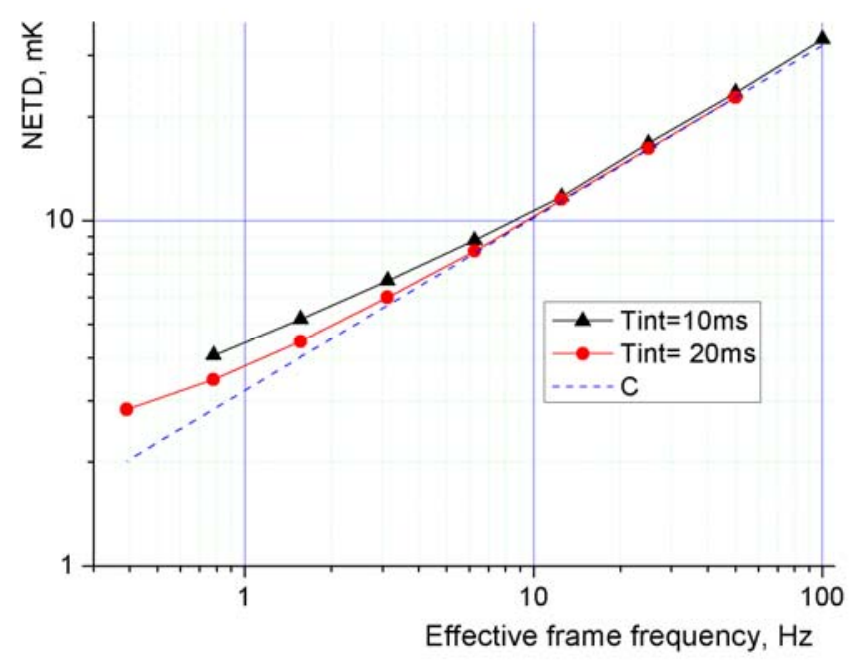

Fig. 6. Temperature resolution dependencies of IR camera TKVr-ISP "SVIT" on efficient frame frequency.

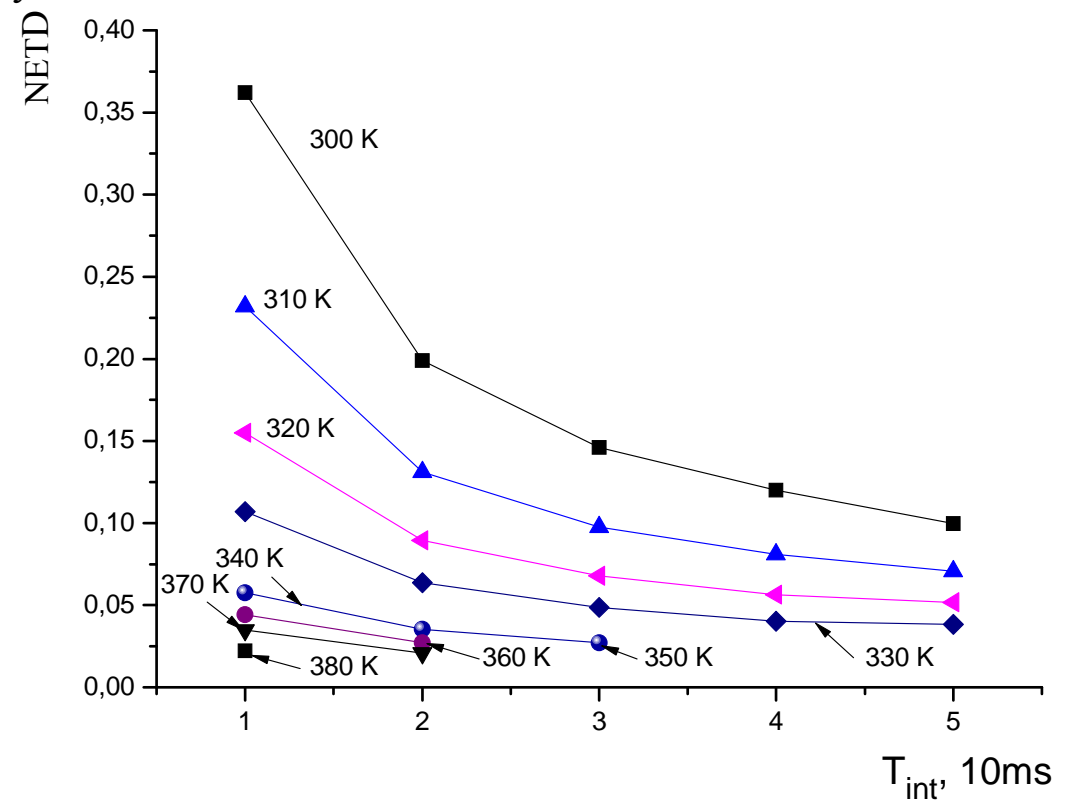

Fig. 7. NETD versus accumulation time for objects of various temperatures. 


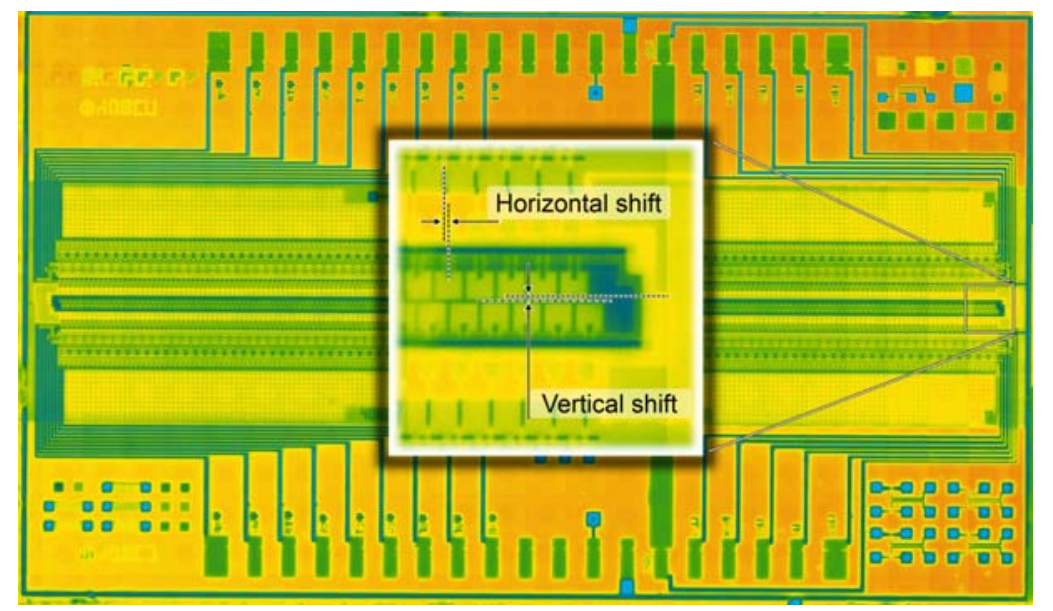

Fig. 8.Control of alignment precision in hybrid modules assembly on In micro-columns. Module sized $6.5 \times 10.5 \mathrm{~mm}$; image dimension 3380x2000 pixels, spatial resolution $\sim 3 \mu \mathrm{m}$, NETD - from $10 \mathrm{mK}$ depending on the temperature of objects under study. An enlarged image fragment is shown in the window. 